\title{
Efecto de la asignatura de educación física en la percepción de los estudiantes frente al desarrollo de competencias genéricas Effect of Physical Education on the students' perception regarding the development of generic competences

\author{
*Jennifer Jones - Jofré, **Sheyla Müller- Pérez ***Fernando Barraza - Gómez \\ *UniversidadTécnica Federico Santa María (Chile), **Universidad San Sebastián (Chile), ***UniversidadViña del Mar
} (Chile)

\begin{abstract}
Resumen. El desarrollo de competencias por medio de la práctica sistemática de la actividad física se ha vuelto un desafío. Por lo anterior, el objetivo de esta investigación es conocer el efecto de las clases de educación física en el desarrollo de competencias genéricas mediante la percepción de los estudiantes, tras la aplicación del Cuestionario de las Competencias Genéricas de Educación Física, participando un total de 961 estudiantes de primer año pertenecientes a las diferentes carreras de la Universidad Técnica Federico Santa María de Valparaíso el año 2017. El Cuestionario fue diseñado para medir la variable de manera exhaustiva, considerando cuatro competencias genéricas: trabajo en equipo, recreación, inteligencia kinestésica y ética, las que forman parte de los programas de asignaturas de educación física. Según los resultados obtenidos se observa que en el caso de las competencias genéricas inteligencia kinestésica y ética, el valor obtenido fue de $\mathrm{p}<0,000$. Para las competencias genéricas trabajo en equipo y recreación el valor fue $\mathrm{p}<0,002$. Asimismo, el cuestionario presenta un $95 \%$ de confianza, evidenciando diferencias significativas, ya que el nivel de p-valor (Sig.) es $\mathrm{p}<0,05$, y el valor obtenido fue de $<0,000$, concluyendo que los estudiantes reconocen que las clases de educación física permiten a través de la práctica sistemática de la actividad física el desarrollo de estas competencias genéricas, contribuyendo así a la formación integral del individuo.

Palabras claves: educación física, competencias genéricas, formación integral, trabajo en equipo, ética, inteligencia kinestésica.
\end{abstract}

\begin{abstract}
The development of skills throughout the systematic practice of Physical Education (PE) has become a challenge. This is the reason why the focus of this reasearch is to know the effects of Physical Education on the students' perception regarding the development of generic competences, applying the Questionnaire of Generic Physical Education Competences USM to 961 first year students from differents USM careers in 2017. The questionaire was designed to measure the variable exhaustively, considering four generic competences: teamwork, recreation, kinesthetic intelligence and ethics. These skills are part of the PE course outlines. The results showed that, in the case of the kinesthetic intelligence and ethics generic competences, the $\mathrm{p}$-value was $\mathrm{p}<0,000$. For the teamwork and recreation generic competences, the value was $\mathrm{p}<0.002$. The Questionnaire holds a $95 \%$ of confidence and shows significant differences, since the significant $p$-value is $p<0,05$ and the obtained value was $\mathrm{p}<0,000$. Therefore, it is concluded that students recognize that PE allow the development of generic skills throughout the systematic practice of physical activity, confirming that physical activity favors the development of generic competences and therefore, and contributes to the integral formation of the student.
\end{abstract}

Keywords: physical education, generic skills, team work, ehics, kinesthetic intelligence, integral formation.

\section{Introducción}

Hoy en día la educación vive momentos de constantee transformaciones afectando a las instituciones de educación superior. Dado lo anterior en los últimos años el Consejo de Rectores de las Universidades Chilenas (CRUCH) ha discutido el tema de llevar a cabo innovaciones curriculares en las universidades, basando dichas innovaciones en un enfoque por competencias (Informe Innovación Curricular en las Universidades del Consejo de Rectores, 2000-2010), incorporando nue-

Fecha recepción: 29-07-21. Fecha de aceptación: 19-11-21

Fernando Barraza - Gómez

ferbarrago@gmail.com vas estrategias de enseñanzas, evaluaciones y también exigiendo competencias docentes, con el fin de mejorar el proceso de enseñanza aprendizaje de los estudiantes (Pugh \& Lozano, 2019).

Por lo anterior es que la Universidad Técnica Federico Santa María (USM) no queda ajena a dichos cambios, actualizando su modelo educativo hacia un enfoque curricular basado en competencias, reflexionando acerca del proceso formativo que compromete a los estudiantes (Ojeda, Carter, Cresp, Sanhueza \& Machuca, 2019) con el fin de responder a los cambios y transformaciones que el sistema educativo va demandando.

Con la implementación de este nuevo modelo educativo, es relevante incorporar competencias transversales (Ojeda et al., 2019). Dado lo anterior, surgen sie- 
te nuevas Competencias Transversales como un sello distintivo de los egresados de la USM. La determinación de estas competencias se forjó a partir de un sondeo general, tanto a los empleadores del medio, profesores, estudiantes y egresados, entre los años 2012-2014. Las competencias seleccionadas responden no solo a aquellas que los estudiantes deben desarrollar durante su proceso de formación profesional, sino que algunas son distintivas de los egresados de la institución, siendo estas: a)responsabilidad social y ética, b)resolución de problemas, c)compromiso con la calidad, d)innovación y emprendimiento, e)manejo de las tecnologías de información y comunicaciones, f)comunicación efectiva y g)vida saludable (Modelo Educativo Universidad Santa María, 2015). Es así como se define la competencia transversal sello Vida Saludable desde la mirada del estudiante que «practica el autocuidado, el autodesarrollo y la autogestión, a través de la actividad física y la vida saludable, para alcanzar un desarrollo humano integral» (Jones \& Hecht, 2016, p.12). Es importante señalar que la USM ha considerado la actividad física, a través de sus múltiples expresiones, como una herramienta importante dentro de la formación integral del estudiante, estando presente desde los primeros programas de formación estudiantil desde el año 1932 (Departamento de Educación Física, Deportes y Recreación, 2017), considerando no solo el desarrollo cognitivo del ingeniero sino potenciando la formación humana integral, es decir, «el proceso por medio del cual se busca el desarrollo armónico de las diferentes potencialidades de la persona» (Tobón, 2010,p.96).

A partir del nuevo modelo educativo institucional, el Departamento de Educación Física, Deportes y Recreación (DEFIDER) comienza a trabajar en la armonización curricular, lo que conlleva a definir ocho competencias genéricas: trabajo en equipo, ética, recreación, ocio, inteligencia kinestésica, tratamiento de conflicto y negociación, responsabilidad social y temple, que serán desarrolladas a partir de los distintos espacios educativos que propicia la unidad docente. En el caso de los programas de asignaturas de educación física, la armonización curricular no solo permitió definir nuevos resultados de aprendizajes sino que además se seleccionaron 4 de las 8 competencias genéricas, definidas en la unidad, para ser desarrolladas a través de las distintas actividades físicas ejecutadas en las clases de las asignaturas, aportando a su vez a la competencia transversal sello Vida Saludable a través del logro de los resultados de aprendizajes y desarrollo de las competencias genéricas, contribuyendo directamente en la formación in- tegral de los estudiantes (Jones \& Hecht, 2016).

Al referirse al término competencias este resulta ser amplio, pudiéndose definir como comportamientos que son capaces de dar respuesta ante determinadas situaciones, respondiendo de forma exitosa (García, 2019), como resultante de los conocimientos, habilidades y también de las actitudes que moviliza una persona (Villarroel \& Bruna, 2017). Por su parte, Corvalán y Hawes (2006) señalan que una competencia integra 3 elementos: conocimientos, procedimientos y actitudes. Asimismo, una competencia se puede definir como la capacidad o habilidad de ejecutar tareas, haciendo frente a distintas situaciones de forma eficaz, movilizando actitudes, habilidades y conocimientos de forma interrelacionada representando una combinación dinámica de estos, resultando fundamental el apoyo de los programas educativos los cuales fomentan el desarrollo de competencias (Guzmán \& Marín, 2011; Roncancio, Mira \& Muñoz, 2017). Del mismo modo la Universidad de Concepción en Chile acoge este concepto, ya que de acuerdo con su rediseño curricular llevado a cabo en sus carreras de pedagogía consideraron la definición de competencias como una combinación dinámica de conocimientos, comprensión, habilidades y capacidades (Cisterna, Soto \& Rojas, 2016).

En el caso de las competencias genéricas (competencias implicadas en esta investigación), se definen como aquellas que están presentes transversalmente en la mayoría de las tareas que deben realizar los profesionales (Hurtado, Reyes, Saavedra \& Zúñiga, 2013), considerándose como fundamentales, ya que se relacionan con distintas materias y campos ocupacionales (Esteban \& Menjívar de Barbón, 2011; Ojeda, Carter, López, Fuentes \& Gallardo, 2022).

Es relevante señalar que en el marco del Espacio Europeo de Educación Superior se han promovido distintas iniciativas con el fin de identificar las competencias tanto de la especialidad como genéricas, así como también la Comisión Nacional de Acreditación en Chile, quien ha levantado algunas competencias genéricas para ser desarrolladas en la educación superior (Pugh \& Lozano, 2019).

Es por lo anterior que la asignatura de educación física está llamada a organizar su currículo, con el fin de favorecer la adquisición de competencias que se relacionen con las distintas acciones motrices que se desarrollen en las clases, integrando esas vivencias a la vida de los estudiantes (Abarca, Murillo, Julián, Zaragoza, \& Generelo, 2015), buscando la forma que transfieran el aprendizaje de las clases a lo largo de la vida dando cuenta 
del desarrollo de competencias. Es así como la asignatura de educación física enfrenta retos en su currículo debido al contexto educativo global, replanteándose interrogantes vinculadas tanto a la finalidad de la asignatura como a cuál es el rol que esta debe tener en la educación formal de los estudiantes, surgiendo tres focos que el currículo de la educación física debiese atender y considerar en su proceso global: el desarrollo físico-motriz, la creación y recreación de la cultura física y por último el desarrollo integral del alumnado, focalizándose este último tanto en el desarrollo de las competencias básicas como a su vez en el desarrollo integral desde, con y a través de la motricidad en los estudiantes (Abarca et al., 2015; López, Pérez, Manrique \& Monjas, 2016).

En el caso de las asignaturas de educación física los estudiantes, de la USM, de las diferentes carreras de pregrado diurno que cursan las asignaturas, puedan desarrollar durante dos semestres académicos de forma sistemática diferentes actividades que les permite a su vez experimentar el trabajo con las competencias genéricas seleccionadas para cada asignatura entendiendo que, la educación física es capaz de promover aprendizajes relevantes que ninguna otra asignatura puede fomentar, los que pueden ser aplicados tanto en el ámbito personal como social (Cañabate \& Zalagaz, 2011), contribuyendo en la formación integral del alumnado, propiciando un espacio educativo formativo para aquello (López et al., 2016). Por lo tanto, el objetivo de este estudio es conocer el efecto de las clases de educación física en el desarrollo de competencias genéricas en los estudiantes, durante dos semestres académicos en el primer año de ingreso a la universidad, mediante la percepción de los estudiantes.

\section{Material y Método}

\section{Diseño}

En la presente investigación se utiliza un análisis estadístico comparativo, pues recoge información de un grupo de estudiantes de primer año que cursaron las asignaturas de educación física, proceso que dura 2 semestres académicos. Además, la investigación responde al diseño no experimental transversal, cuya población a analizar fueron todos los estudiantes de las carreras de pregrado diurna de primer año de la USM casa central, de Valparaíso.

\section{Muestra}

De un total de 1254 estudiantes de primer año, participaron 961estudiantes que representa a un 76,6\% de la población total, pertenecientes a las diferentes carreras de pregrado diurna que se dictaban en el campus casa central de la USM de Valparaíso, los cuales respondieron de manera libre y voluntaria en cada aplicación del cuestionario mediante el aula virtual de la asignatura, sitio donde se encontraba el cuestionario, resguardando la identidad de los participantes, respondiendo a una muestra no probabilística casual.

\section{Instrumento}

Con el fin de conocer el efecto de las clases de edu-

Tabla 1

Cuestionario de las competencias genéricas de educación físca de tusm.

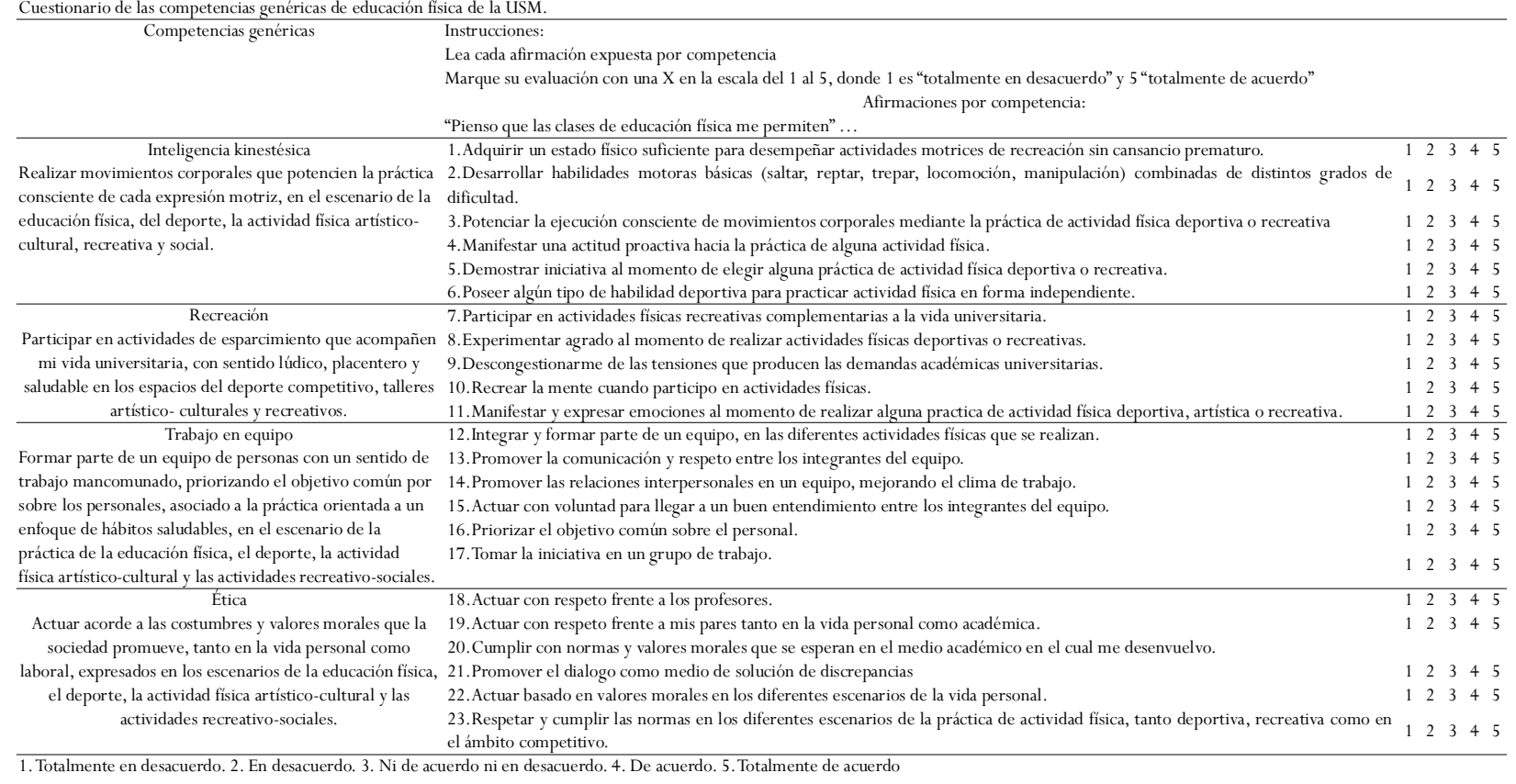


cación física en el desarrollo de las 4 competencias genéricas, se diseñó y aplicó el Cuestionario de Competencias Genéricas de Educación Física. El cuestionario constó de 23 preguntas, agrupadas en 4 dimensiones que representan cada una de las competencias genéricas: la primera responde a la competencia genérica Inteligencia Kinestésica, que tiene seis afirmaciones asociadas; la segunda es la competencia genérica Recreación, que tiene cinco afirmaciones; luego está la competencia genérica Trabajo en Equipo, la cual tiene asociada seis afirmaciones; y, por último, está la competencia genérica Ética, que tiene asociada seis afirmaciones. El mecanismo para responder fue por Escala Likert con 5 intenciones donde $1=$ totalmente en desacuerdo; $2=$ en desacuerdo; $3=$ ni de acuerdo ni en desacuerdo; $4=$ de acuerdo y $5=$ totalmente de acuerdo (tabla 1 ).

\section{Validación del instrumento}

Para poder llevar a cabo el análisis estadístico fue necesario realizar una validación del cuestionario, aplicando procesos de validación que han sido utilizados en recientes estudios (Estrada, Vega, Castillo, Müller, \& Boada, 2021). En primera instancia se validó el contenido participando 4 expertos que conocían tanto el modelo educativo institucional como también el DEFIDER, elaborando y seleccionando ítems que permitieran medir la variable de manera exhaustiva. Para lo anterior se envió el cuestionario a los expertos, junto a material de apoyo acorde al constructo del cuestionario (definiciones del modelo educativo, modelo pedagógico del DEFIDER y definiciones de las competencias genéricas). De esta forma los expertos realizaron su retroalimentación, en relación con las afirmaciones propuestas en el cuestionario, aplicando los cambios sugeridos para posteriormente validar el instrumento empleando: el análisis factorial confirmatorio (CFA, mediante el uso del Software Amos), una validación de consistencia interna de los ítems que forman cada dimensión del cuestionario e identificando el ajuste del modelo global. La consistencia interna se comprueba mediante la validez convergente, la cual busca tanto que los ítems sean representativos a la dimensión que se vinculan como también que exista fiabilidad de consistencia interna de cada dimensión, es decir, que identifique el grado de correlación entre los ítems de cada dimensión o competencia genérica, y la validez discriminante que asegura que los ítems de cada constructo solo aporten a esa dimensión y no a otras dimensiones. De acuerdo con los resultados solamente la dimensión o competencia genérica inteligencia kinestésica no cum- plió con la validez convergente por lo que se elimina el ítem 1 como resultado de lo anterior (tabla 2). Luego se estima otra vez el instrumento, resultando que los ítems 7, 11 y 17, de las competencias inteligencia kinestésica, recreación y trabajo en equipo respectivamente, no cumplen con el criterio de comunalidad mayor a 0.5 , pero siguiendo las recomendaciones de Hair et al. (2019), se decide mantenerlos ya que los ítems resultan ser importantes para el instrumento y presentan

\begin{tabular}{|c|c|c|c|c|c|c|}
\hline \multirow{2}{*}{ Dimensión } & \multirow{2}{*}{ Indicador } & \multicolumn{3}{|c|}{ Validez Convergente } & \multicolumn{2}{|c|}{ Fiabilidad de Consistencia Interna } \\
\hline & & Carga & Comunidad & AVE & Alpha de Cronbach & $\mathrm{CR}$ \\
\hline \multirow{7}{*}{$\begin{array}{l}\text { Inteligencia } \\
\text { Kinestésica }\end{array}$} & *Q01_1 & 0.59 & 0.35 & 0.49 & 0.86 & 0.87 \\
\hline & Q02_2 & 0.73 & 0.53 & & & \\
\hline & Q03_3 & 0.75 & 0.57 & & & \\
\hline & Q04_4 & 0.72 & 0.52 & & & \\
\hline & Q05 5 & 0.72 & 0.51 & & & \\
\hline & Q06_6 & 0.74 & 0.55 & & & \\
\hline & Q07_7 & 0.62 & 0.38 & 0.53 & 0.83 & 0.84 \\
\hline \multirow{4}{*}{ Recreación } & Q08_8 & 0.78 & 0.61 & & & \\
\hline & Q09_9 & 0.74 & 0.55 & & & \\
\hline & Q10_10 & 0.79 & 0.63 & & & \\
\hline & Q11_11 & 0.69 & 0.48 & & & \\
\hline \multirow{6}{*}{$\begin{array}{l}\text { Trabajo en } \\
\text { equipo }\end{array}$} & Q12_12 & 0.77 & 0.59 & 0.58 & 0.89 & 0.89 \\
\hline & Q13_13 & 0.78 & 0.61 & & & \\
\hline & Q14_14 & 0.81 & 0.66 & & & \\
\hline & Q15_15 & 0.80 & 0.65 & & & \\
\hline & Q16_16 & 0.71 & 0.50 & & & \\
\hline & Q17_17 & 0.68 & 0.47 & & & \\
\hline \multirow{6}{*}{ Ética } & Q18_18 & 0.84 & 0.71 & 0.67 & 0.92 & 0.92 \\
\hline & Q19_19 & 0.86 & 0.73 & & & \\
\hline & Q20_20 & 0.82 & 0.67 & & & \\
\hline & Q21_21 & 0.81 & 0.65 & & & \\
\hline & Q22_22 & 0.79 & 0.63 & & & \\
\hline & Q23_23 & 0.80 & 0.64 & & & \\
\hline \multicolumn{7}{|c|}{ *: item 1 de la competencia inteligencia kinestésica } \\
\hline \multicolumn{7}{|c|}{ Tabla 3.} \\
\hline \multicolumn{7}{|c|}{ Resultados segunda aplicación del Análisis Factorial Confirmatorio } \\
\hline Din & Jadiod & Vali & dez Convergel & & Fiabilidad de Consister & Interna \\
\hline Dimension & Indicador & Carga & Comunalida & ad AVE & Alpha de Cronbach & $\mathrm{CR}$ \\
\hline & Q02_2 & 0.72 & 0.50 & 0.51 & 0.854 & 0.86 \\
\hline & Q03_3 & 0.75 & 0.55 & & & \\
\hline Inteligencia & Q04_4 & 0.74 & 0.54 & & & \\
\hline Kinestésica & Q05_5 & 0.73 & 0.52 & & & \\
\hline & Q06_6 & 0.73 & 0.54 & & & \\
\hline & $*$ Q07_7 & 0.62 & 0.39 & 0.53 & 0.831 & 0.839 \\
\hline & Q08_8 & 0.78 & 0.61 & & & \\
\hline D & Q09_9 & 0.74 & 0.55 & & & \\
\hline Recreacion & Q10_10 & 0.79 & 0.63 & & & \\
\hline & *Q11_11 & 0.69 & 0.48 & & & \\
\hline & Q12_12 & 0.77 & 0.59 & 0.58 & 0.886 & 0.891 \\
\hline & Q13_13 & 0.78 & 0.61 & & & \\
\hline Trabajo en & Q14_14 & 0.81 & 0.66 & & & \\
\hline equipo & Q15_15 & 0.80 & 0.65 & & & \\
\hline & Q16_16 & 0.71 & 0.50 & & & \\
\hline & *Q17_17 & 0.68 & 0.47 & & & \\
\hline & Q18_18 & 0.84 & 0.71 & 0.67 & 0.923 & 0.924 \\
\hline & Q19_19 & 0.86 & 0.73 & & & \\
\hline Étic & Q20_20 & 0.82 & 0.67 & & & \\
\hline & Q21_21 & 0.81 & 0.65 & & & \\
\hline & Q22_22 & 0.79 & 0.63 & & & \\
\hline & Q23_23 & 0.80 & 0.64 & & & \\
\hline $\begin{array}{l}* \text { : ítems que } \\
\text { dado que pres }\end{array}$ & $\begin{array}{l}\text { o cumplen c } \\
\text { sentan una val }\end{array}$ & $\begin{array}{l}\text { on el crite } \\
\text { lidez de st }\end{array}$ & $\begin{array}{l}\text { rio de comun } \\
\text { contenido y }\end{array}$ & $\begin{array}{l}\text { nalidad } \mathrm{m} \\
\text { un valor }\end{array}$ & $\begin{array}{l}\text { nayor a } 0,05 \text {, pero se ma } \\
\text { r? } 0,05 \text { del AVE. }\end{array}$ & \\
\hline Tabla 4. & & & & & & \\
\hline Validación Cor & nvergente del $\mathrm{C}$ & uestionaric & & & & \\
\hline Tipo de Valid & lación & & & & Criterio & \\
\hline Validez Conver & ggente & & & & & \\
\hline Carga Factor & & & & & $=0.4$ & \\
\hline Comunalida & & & & & $>0.5$ & \\
\hline Varianza exts & aída promedi & io (AVE) & & & $>0.5$ & \\
\hline Fiabilidad de & Consistencia & & & & & \\
\hline Fiabilidad co & mpuesta $(\mathrm{CR}$ & & & & $>0.7$ & \\
\hline Alfa de Cron & bach & & & & $>0.7$ & \\
\hline $\begin{array}{l}\text { Tabla } 5 . \\
\text { Medidas Glol }\end{array}$ & bales. & & & & & \\
\hline Ratio de ?2 & & 2.50 & & No tie & ene buen ajuste & \\
\hline SRMR & & 0.04 & & Buen $A$ & Ajuste & \\
\hline RMSEA & & 0.06 & & Ajuste & Aceptable & \\
\hline TLI & & 0.95 & & Ajuste & Aceptable & \\
\hline CFI & & 0.94 & & Ajuste & Aceptable & \\
\hline
\end{tabular}


una validez de su contenido, sumado a que el AVE de la dimensión es mayor a 0.5 (tabla 3). Al mismo tiempo, se realizó la validación convergente resultando que todas las dimensiones cumplieron con esta validación (tabla 4). Para cada validación se siguieron los criterios establecidos por Aldás y Uriel (2017) y Hair et al. (2005). Asimismo, en la tabla 5 se puede observar que el cuestionario cumple con un ajuste global aceptable, ya que el SRMR es de 0.04 y el error cuadrático medio raíz de aproximación (RMSEA) es de 0.06.

\section{Procedimiento}

El instrumento utilizado buscaba evidenciar la existencia de un desarrollo de las competencias genéricas a través de la práctica sistemática de la actividad física realizadas en las asignaturas de educación física. Dichas asignaturas se dictan en el primer año de los estudiantes, cuya duración es de 17 semanas académicas semestrales, para cada una, desarrollándose una vez a la semana, cuyo contacto con los estudiantes fue de 1 hora y media de clases, cuantificando el programa de cada asignatura con dos SCT. En cuanto a la aplicación del cuestionario, se desarrolló en dos momentos del año académico de los estudiantes que cursaban las asignaturas de educación física. La primera aplicación se llevó a cabo a inicios del primer semestre, donde participaron 536 estudiantes, mientras que en la segunda aplicación fue realizada a fines del segundo semestre académico, respondiendo 425 estudiantes. En cuanto a los estudiantes que respondieron tanto la primera como segunda aplicación del cuestionario suman un total de 222 estudiantes. Todos los estudiantes cursaban el primer año de universidad y pertenecían a las diferentes carreras de pregrado diurna que dicta la USM en el campus Valparaíso. Por último, cabe señalar que el área docente del DEFIDER autorizó la aplicación del instrumento a los estudiantes que cursaban las asignaturas de educación física (tanto en el primer como segundo semestre), con la única finalidad de contribuir a la formación integral de los estudiantes.

\section{Resultados}

De acuerdo con los resultados, el instrumento para medir competencias genéricas cumple con los criterios que establece el análisis factorial confirmatorio (CFA) de consistencia y convergencia, por lo anterior podemos afirmar que el instrumento es válido y confiable para realizar las estimaciones requeridas.

En relación con la aplicación del instrumento, en la tabla 6 se evidencia el número de estudiantes que participaron en el pre y post test. No obstante, para el análisis de los resultados, se consideró el $\mathrm{n}=222$ participantes, correspondientes a aquellos estudiantes que participaron de todo el proceso. En la tabla 7 se observa que en el pre test la competencia genérica trabajo en equipo logra la media mayor $(4,38 \pm 0,50)$, obteniendo la media menor la competencia genérica inteligencia kinestésica con un valor de 4,24 $\pm 0,50$. En el post test del cuestionario, la media mayor es lograda por la competencia genérica ética $(4,52 \pm 0,52)$, acercándose al puntaje máximo del instrumento (5 puntos), mientras que la competencia inteligencia kinestésica nuevamente evidencia una media menor $(4,39 \pm 0,47)$.

En cuanto a los resultados del pre test los estudiantes reconocen que la asignatura de educación física desarrolla la competencia trabajo en equipo, ya que les permite: a) integrar y formar parte de un equipo, en las diferentes actividades físicas que se realizan; b) promover la comunicación y respeto entre los integrantes del equipo; c) promover las relaciones interpersonales en un equipo, mejorando el cima de trabajo; d) actuar con voluntad para llegar a un buen entendimiento entre los integrantes del equipo; e) priorizar el objetivo Común sobre el personal y f) tomar la iniciativa en un grupo de trabajo. Asimismo, tras los resultados del post test los estudiantes afirman que desarrollan la competencia genérica ética en la asignatura de educación física, ya que las actividades ejecutadas les permite: a) ac-

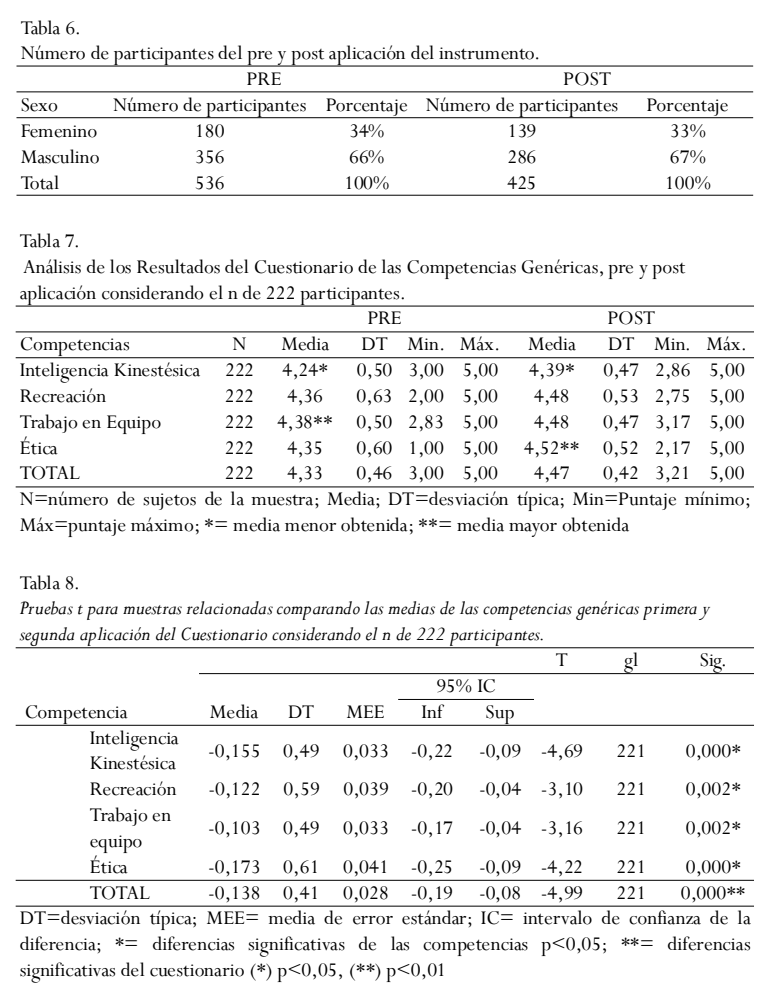


tuar con respeto frente a los profesores; b) actuar con respeto frente a mis pares tanto en la vida personal como académica; c) cumplir con normas y valores morales que se esperan en el medio académico en el cual me desenvuelvo; d) promover el dialogo como medio de solución de discrepancias; e) actuar basado en valores morales en los diferentes escenarios de la vida personal y f) respetar y cumplir las normas en los diferentes escenarios de la práctica de actividad física, tanto deportiva recreativa como en el ámbito competitivo.

Sumado a lo anterior, la tabla 8 evidencia que el cuestionario presenta un 95\% de confianza y tras la aplicación de una prueba $t$ para muestras relacionadas se observa que existen diferencias significativas referente a las competencias genéricas, que en el caso de las competencias genéricas inteligencia kinestésica y ética el valor obtenido fue de $\mathrm{p}<0,000$. Para las competencias genéricas trabajo en equipo y recreación el valor fue $\mathrm{p}<0,002$. Asimismo, el cuestionario en general demuestra diferencias significativas ya que el nivel de $\mathrm{p}$-valor (Sig.) es $\mathrm{p}<0,05$ cuyo valor obtenido fue de $<0,000$. Lo anterior resulta relevante ya que permite analizar la percepción de los estudiantes frente al proceso educativo enfocado en competencias, confirmando que las actividades físicas desarrolladas en clases propician el desarrollo de las competencias genéricas, contribuyendo así al desarrollo integral de los estudiantes.

\section{Discusión}

De acuerdo con los resultados obtenidos tanto en la primera como en la segunda aplicación del cuestionario, se evidencia que las 4 competencias genéricas evaluadas en las clases de las asignaturas de educación física incrementan su valor, resultando la competencia trabajo en equipo (pre test) y ética (post test) las que registraron una media más cercana al máximo otorgado en el cuestionario ( 5 puntos).

En relación con la competencia genérica trabajo en equipo, que logra la media mayor en la primera aplicación del cuestionario, se puede afirmar que las actividades físicas desarrolladas en las clases propician el trabajo entre dos o más personas, que interactúan entre sí siendo relevante que tengan uno o más objetivos comunes, donde además cada individuo tiene roles y responsabilidades (Saraswat \& Khandelwal, 2015). Tal como lo señala la definición de la competencia genérica trabajo en equipo, el trabajo mancomunado y la priorización de los objetivos comunes por sobre los personales resultan claves para el desarrollo de esta competencia (Jones \&
Hecht, 2016), resultando el concepto de objetivo común como un medio para que el equipo pueda ser eficaz y logre alcanzar una determinada meta, diferenciándolos de otros equipos que simplemente manifiestan ser un grupo. Al mismo tiempo, el concepto de cooperación se relaciona con el trabajo en equipo ya que permite a los integrantes de algún equipo deportivo trabajar en las relaciones interpersonales, reforzando la idea de alcanzar una meta en común en la cual todo el equipo haya sido partícipe de lograr dicho resultado (García-Mas, 2001; López \& Taveras, 2022).

Sumado a lo anterior, el concepto cohesión es considerado, como uno de los elementos claves para la interacción dentro del equipo, rescatando también el concepto de coordinación ya que al momento de interactuar y realizar acciones motoras requieren de una coordinación como también de la comunicación, siendo este importante al momento de mejorar la cooperación en el trabajo en equipo, sumado a cualidades tales como la lealtad y resolución de problemas, permitiendo a los estudiantes lidiar con ciertos conflictos y por ende poder resolverlos (García-Mas, 2001; Saraswat \& Khandelwal, 2015).

En cuanto a la competencia genérica ética, la cual destaca con una media mayor en la segunda aplicación del cuestionario, esta se relaciona no solo a actuar acorde a las costumbres que la sociedad promueve, sino que también a los valores morales que está también fomenta (Jones \& Hecht, 2016). De acuerdo con algunos autores, hoy en día el deporte es parte de la formación de los niños, siendo una herramienta útil para la entrega de valores, recibiendo una formación y educación integral basada en valores que se vinculan con el deporte (Ginesta, 2007). Si llevamos la competencia ética al escenario de la asignatura de educación física, esta se sustenta en la promoción de comportamiento éticos y morales, promoviendo actividades que estimulen la mejora en las conductas de los estudiantes (Planchuelo, 2008). Por lo tanto, a través del proceso de enseñanza aprendizaje vivido en la asignatura de educación física, los estudiantes pueden adquirir valores a través de la práctica de la actividad física o ejercicio físico desarrollado durante las clases (Arufe, 2011). Por otra parte, el rol de profesor de educación física es muy relevante dentro de la clase, ya que es capaz de transferir valores a través de la práctica de actividad física o deportiva (Pezdek, 2012). Por lo tanto, el profesor de educación física tiene la responsabilidad a través de sus clases educar a los estudiantes (Beregüí \& Garcés, 2007).

Por último, las competencias genéricas recreación e 
inteligencia kinestésica que, si bien evidenciaron una media menor en relación con las competencias genéricas trabajo en equipo y ética de todas formas se observaron cambios significativos, reconociendo que la asignatura de educación física actúa como un medio para el desarrollo de ambas competencias en los estudiantes. En el caso de la competencia genérica inteligencia kinestésica, los autores hacen referencia a que presenta características que pueden ser observadas como la relación con el control del movimiento y el manejo de objetos (De la Cruz Ordoñez \& Cruzata, 2017). Lo anterior se relaciona con la competencia genérica inteligencia kinestésica ya que su definición señala que los movimientos corporales deben realizarse de forma consciente en cada expresión motriz (Jones \& Hecht, 2016). Asimismo, la inteligencia kinestésica considera el uso del cuerpo con el fin de realizar acciones motrices que permitan resolver problemas y también expresar ideas, siendo la asignatura de educación física una herramienta de constante aprendizaje para los estudiantes, ya que permite el desarrollo motor de las personas (Fernández \& Mihura de Rosa, 2015; Arias, Rodríguez, Guerra \& Muelas, 2019).

En cuanto a la competencia genérica recreación, se puede vincular como aquella acción que permite que las personas experimenten placer al momento de realizar alguna actividad en el tiempo libre, contribuyendo tanto a su desarrollo integral como al alma de las personas, abarcando su mente y a su vez el desarrollo físico de su musculatura (Meneses \& Monge, 1999; Yfanti, Samara, Kazantzidis, Hasiotou \& Alexiou, 2014), relacionándose justamente con la definición de la competencia genérica recreación, la cual rescatar el participar en actividades de esparcimiento, pero que además tengan «un sentido lúdico, placentero y saludable» (Jones \& Hecht, 2016, p.13). Es por todo lo anterior que se plantea que la recreación físico-deportiva se ha convertido en una de las actividades que más se practica en la sociedad en el tiempo libre, brindando a las personas la oportunidad de disfrutar de su tiempo libre, entretenerse y además experimentar algunas relaciones interpersonales, sumado a sensaciones de placer (Molina \& Valenciano, 2010).

\section{Conclusiones}

A partir de los resultados obtenidos se puede concluir que los estudiantes reconocen que las asignaturas de educación física permiten el desarrollo de las 4 competencias genéricas contenidas en sus programas: tra- bajo en equipo, recreación, inteligencia kinestésica y ética, gracias a la práctica de las actividades planteadas y desarrolladas en las clases, logrando un efecto positivo en el desarrollo de las competencias, existiendo una diferencia significativa en los resultados obtenidos por los estudiantes entre la primera y segunda aplicación del cuestionario. Según los estudiantes, las competencias genéricas que más se logran desarrollar fueron trabajo en equipo y ética, de acuerdo con los resultados obtenidos en la primera y segunda aplicación del cuestionario respectivamente.

En este sentido resulta relevante que las asignaturas de educación física a través de las actividades ejecutadas logren desarrollar las competencias genéricas, centrando su proceso educativo en los estudiantes para que así puedan vivenciar las distintas acciones propiciadas en clases. Por lo anterior, es relevante conocer las percepciones de los estudiantes en relación con el proceso educativo vivenciado en las clases ya que entregan información respecto a que las actividades desarrolladas en las asignaturas de educación física propician un espacio educativo que permite el desarrollo de las competencias genéricas, contribuyendo a la formación integral de los estudiantes. Asimismo, se evidencia un desarrollo significativo en las competencias genéricas, sobre todo luego de haber cursado el segundo semestre, ya que todas las competencias genéricas obtuvieron una media mayor tras la segunda aplicación del cuestionario, lo que demuestra que la práctica sistemática de la actividad física realizada durante 2 semestres produce cambios positivos en los comportamientos de los estudiantes que resultan en un reconocimiento de la importancia del desarrollo de las 4 competencias genéricas declaradas en los programas de asignaturas, contribuyendo tanto al logro de los resultados de aprendizajes como a la competencia transversal sello Vida Saludable.

Los resultados de este estudio pueden ser de interés para las instituciones de educación superior que imparten asignaturas de educación física en las mallas curriculares de los estudiantes, como contribución a la formación integral de los futuros profesionales.

\section{Referencias}

Abarca,A., Murillo, B., Julián, J., Zaragoza, J., \& Generelo, E. (2015). La Educación Física ¿Una Oportunidad Para La Promoción De LaActividad Física? Retos, 28, 155-159 doi: https: / / zaguan.unizar.es/record/70302/files/texto_completo.pdf

Aldás, J., \& Uriel,E. (2017).Análisis multivariante aplicado con R.Análisis MultivarianteAplicado Con R. Madrid: Ediciones Paraninfo, SA. 
Molina, J., \& Valenciano, J. (2010). La recreación físico-deportiva y su tratamiento del cuerpo: Un análisis crítico. Apuntes Educación Física y Deportes, 100, 66-72 doi: https: / /www.redalyc.org/pdf/5516/ 551656926008.pdf

Arias, G., Rodríguez,A., Guerra, N., \& Muelas, M., (2019). Las inteligencias múltiples y su expresión desde la kinestésica-corporal en la educación física. Revista Científica Olimpia, 16(57), 57-65 doi: https: / / revistas.udg.co.cu/index.php/olimpia/article/view/1156

Arufe,V.(2011). La educación en valores en el aula de educación ¿mito o realidad? Emás F: Revista Digital De Educación Física, 9, 32-42 doi: https: / / dialnet.unirioja.es/servlet/articulo?codigo $=3618413$

Beregüí, R., \& Garcés, E. (2007).Valores en el deporte escolar: Estudio con profesores de educación física. Cuadernos de Psicología del Deporte, 7, 90-103 doi: https://revistas.um.es/cpd/article/view/54621

Cañabate, D., \& Zagalaz, M. L. (2011). Aportaciones de la educación física al desarrollo de la competencia básica: Autonomía e iniciativa personal.Tándem: Didáctica de la Educación Física, 37, 69-77.

Cisterna, C., Soto,V., \& Rojas, C. (2016). Rediseño curricular en la universidad de Concepción: La experiencia de las carreras de formación inicial docente. Calidad en la Educación, 44, 301-323 doi: https:// scielo.conicyt.cl/scielo.php?pid=S07 18 45652016000100011\&script $=$ sci_arttext\&tlng $=\mathrm{n}$

Corvalán, O., \& Hawes, G. (2006). Aplicación del enfoque de competencias en la construcción curricular de la universidad de Talca, Chile. Revista Iberoamericana de Educación, 40(1), 1-17 doi: https:// rieoei.org/historico/deloslectores/1463Corvalan.pdf

De la Cruz Ordoñez,A., \& Cruzata,A. (2017). Inteligencia emocional y kinestésica en la educación física de la educación primaria. Actualidades Investigativas en Educación, 17(2), 1-20 doi: https:// www.scielo.sa.cr/scielo.php?pid=S1409. 47032017000200262\&script=sci_arttext

Departamento deEducación Física, Deportes y Recreación, 2017, Modelo Pedagógico, UniversidadTécnica Federico Santa María, Chile.

Esteban, R., \& Menjívar, S. (2011). Una mirada internacional a las competencias docentes universitarias: Investigación en primera persona: Profesores y estudiantes. Barcelona: Ediciones Octaedro- ICE-UB doi: https://www.torrossa.com/en/resources/an/2914614

Estrada, C.,Vega,A., Castillo,D., Müller,S., \& Boada,J.(2021).Technostress of ChileanTeachers in the Context of the COVID-19 Pandemic and Teleworking. International journal of environmental researchand public health, 18(10), 5458 doi: https:/ /www.mdpi.com/1660-4601/ $18 / 10 / 5458$

Fernández, A., \& Mihura de Rosa, D. (2015). Inteligencias múltiples. Revista deEducación, Motricidad e Investigación, 4, 6-17.

Garcia-Mas, A. (2001). Cooperación y competición en equipos deportivos: Un estudio preliminar. Análisis Psicológica, 19(1), 115-130 doi http://publicacoes.ispa.pt/index.php/ap/article/view/347/pdf

García, M. (2019). Desarrollo de la competencia transversal de aprendizaje autorregulado en estudiantes adultos doi: http:/ /hdl.handle.net/ $11285 / 633029$

Ginesta, X. (2007). Los valores en el deporte: Una experiencia educativa a través del Barça-Madrid. Revista Científica de Comunicación y Educación, 14(28), 148-156 doi:https: / /www.revistacomunicar.com/ ojs/index.php/comunicar/article/view/C28-2007-18

Guzmán, I., \& Marín, R. (2011). La competencia y las competencias docentes: Reflexiones sobre el concepto y la evaluación. Revista Electrónica Interuniversitaria de Formación Del Profesorado, 14(1), 151163.

Hair,J., Black,W., Babin, B.,Anderson, R., yTatham, R. (2005). Multivariate data análisis. London: Prentice-Hall International.
Hurtado, M., Reyes, C., Saavedra, O. \& Zúñiga M. (2016). Competencias Transversales: En la Formación de profesionales de la Universidad Técnica Federico Santa María. Chile. Morfológica.

Informe Innovación Curricular en las Universidades del Consejo de Rectores, 2000-2010 doi:http://vra.ucv.cl/ddcyf/wp-content/uploads/ 2013/06/Informe_innovacion_curricular.pdf

Jones, J.\& Hecht, G. (2016). Competencias Genéricas: asociadas a la actividad física. Departamento de Educación Física, Deportes y Recreación, UniversidadTécnica Federico Santa María, Chile.

López, V., Pérez, D., Manrique, J., \& Monjas, R. (2016). Los retos de la educación física en el siglo XXI. Retos, 29, 182-187 doi https: / / www.redalyc.org/pdf/3457/345743464037.pdf

López, R., \&Taveras, J. (2022). Uso del aprendizaje cooperativo en educación física y su relación con la responsabilidad individual en estudiantes del nivel secundario. Retos: nuevas tendencias en educación física, deporte y recreación, (43), 1-9.

Meneses, M., \& Monge, M. (1999).Actividad física y recreación. Revista Costarricense de Salud Pública, 8(15), 16-24 doi: https:// www.scielo.sa.cr/scielo.php?script $=$ sci_arttext\&pid=S1409. 14291999000200003

Modelo Educativo (2015). UniversidadTécnica Federico Santa María. Chile doi: https://www.usm.cl/descargas/modelo-educativo/ Modelo-Educativo-Institucional-USM.pdf

Ojeda, R., Carter B., Cresp, M., Sanhueza, S., \& Machuca, C. (2019). Evaluación de competencias genéricas en estudiantes de Educación Física: una experiencia en contextos no formales. Retos: nuevas tendencias en educación física, deporte y recreación, (36), 220-227 doi: https: / / dialnet.unirioja.es/servlet/articulo?codigo $=7260906$

Ojeda, R., Carter, B., Pastor, López,V., Fuentes, F., \& Gallardo, F. (2022). Evaluación de competencias genéricas en profesores de Educación Física. Retos: nuevas tendencias en educación física, deporte y recreación, (43), 521-532.

Pezdek, K. (2012). Physical education as a medium of communicating moral values. Acta Universitatis Carolinae: Kinanthropologica,48(1), 126-133 doi: https: / / karolinum.cz/casopis/auc-kinanthropologica/ rocnik-48/cislo-1/clanek-742

Planchuelo, L. (2008). Intervención y evaluación del desarrollo moral en educación física en primaria. Tesis de Doctoral. Departamento de Psicología Social,Antropología Social, Trabajo Social y Servicios Sociales, Facultad de Psicología, Universidad de Málaga, España.

Pugh, G \& Lozano,A (2019). El desarrollo de competencias genéricas en la educación técnica de nivel superior: un estudio de caso. Calidad en la educación, (50), 143-170. https://dx.doi.org/10.31619/ caledu.n50.725

Roncancio, D., Mira, G., \& Muñoz, N. (2017). Las competencias en la formación del profesional contable: Una revisión de las posturas institucionales y educativas en Colombia. Revista Facultad de Ciencias Económicas: Investigación y Reflexión, 25(2), 83-103 doi http:/ /www.scielo.org.co/pdf/rfce/v25n2/0121-6805-rfce-25-02-00083

Saraswat, N., \& Khandelwal, S. (2015). Impact of team building exercises on team effectiveness. International Journal of Marketing and Human Resource Management, 6(3), 89-97.

Tobón, S. (2010). Formación integral y competencias: Pensamiento complejo, currículo, didáctica y evaluación. Colombia. EcoeEdiciones.

Villarroel,V., \& Bruna,D. (2017). Pedagogical Competencies of University Teachers:A Case Study,Which Incorporates the Perspective of Chilean Teachers and Students. Formación universitaria, 10(4), 75-96.https:/ /dx.doi.org/10.4067/S0718-50062017000400008

Yfanti, M., Samara, A., Kazantzidis, P., Hasiotou,A., \& Alexiou, S. (2014). Swimming as physical activity and recreation for women.TIMS, 8(2), 137-145. 